\title{
Competencias TIC de los docentes de matemáticas en el marco del modelo TPACK: valoración desde la perspectiva de los estudiantes ${ }^{1}$
}

\author{
ICT Competencies of Mathematics Teachers' within the Framework \\ of the TPACK Model: Assessment from the Students' Perspective
}

Recibido: 22 de agosto de 2017 - Revisado: 01 de febrero de 2018 - Aceptado: 25 de junio de 2018

\author{
Mayra Alejandra Arévalo Duarte ${ }^{2}$ \\ Miguel Ángel García García ${ }^{3}$ \\ César Augusto Hernández Suárez ${ }^{4}$
}

\section{Resumen}

El modelo TPACK pretende agrupar los tipos de conocimientos que los docentes necesitan dominar para integrar de una manera adecuada las TIC en el aula de clase. El objetivo del artículo fue conocer la percepción de los estudiantes frente a las competencias TIC de los docentes de matemáticas en el contexto del marco TPACK. Se aborda desde el enfoque cuantitativo con un nivel de análisis descriptivo-comparativo que permitió valorar las dimensiones de conocimiento según el modelo TPACK. Como resultados, se describen las relaciones que se establecen de acuerdo a los dominios de conocimiento TPACK las cuales facultan la comprensión del nivel de competencias del docente de matemáticas en relación con la integración de las TIC en su práctica educativa. Se enfatiza en la importancia del conocimiento tecnológico y su articulación en el contexto del aula para su uso y aplicación en el proceso de enseñanza y aprendizaje de las matemáticas.

\section{Palabras clave}

Conocimiento tecnológico pedagógico del contenido - TPACK, Competencias del docente, TIC, Práctica pedagógica, Enseñanza de las matemáticas, Estudiante.

\begin{abstract}
The TPACK model intends to group the types of knowledge that teachers need to master in order to integrate ICT into the classroom appropriately. The article aimed to know the students' perception of the ICT competencies of mathematics teachers in the context of the TPACK framework. The study used the quantitative approach with a descriptivecomparative analysis, which allowed to assess the dimensions of knowledge according to the TPACK model. Regarding results, the description of relationships established according to the TPACK knowledge domains enable the comprehension of the level of competencies of the mathematics teacher concerning the integration of ICT in their educational practice. Emphasis was placed on the importance of technological knowledge and its articulation in the classroom within the process of teaching and learning mathematics.
\end{abstract}

\section{Keywords}

Technological Pedagogical Content Knowledge - TPACK, teacher competencies, ICT, Pedagogical practice, mathematics teaching, Student.

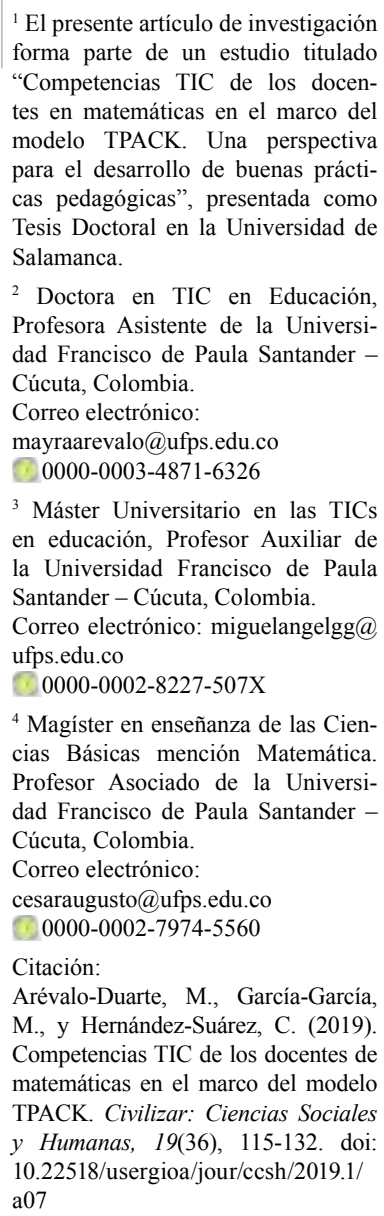

${ }^{1}$ El presente artículo de investigación forma parte de un estudio titulado "Competencias TIC de los docentes en matemáticas en el marco del modelo TPACK. Una perspectiva para el desarrollo de buenas prácticas pedagógicas", presentada como Tesis Doctoral en la Universidad de Salamanca.

2 Doctora en TIC en Educación, Profesora Asistente de la Universidad Francisco de Paula Santander -

Cúcuta, Colombia.

Correo electrónico:

mayraarevalo@ufps.edu.co

0000-0003-4871-6326

${ }^{3}$ Máster Universitario en las TICs en educación, Profesor Auxiliar de la Universidad Francisco de Paula Santander - Cúcuta, Colombia.

Correo electrónico: miguelangelgg@ ufps.edu.co

0000-0002-8227-507X

${ }^{4}$ Magíster en enseñanza de las Ciencias Básicas mención Matemática. Profesor Asociado de la Universidad Francisco de Paula SantanderCúcuta, Colombia.

Correo electrónico:

cesaraugusto@ufps.edu.co

0000-0002-7974-5560

Citación:

Arévalo-Duarte, M., García-García, M., y Hernández-Suárez, C. (2019). Competencias TIC de los docentes de matemáticas en el marco del modelo TPACK. Civilizar: Ciencias Sociales y Humanas, 19(36), 115-132. doi: 10.22518/usergioa/jour/ccsh/2019.1/ $\mathrm{a} 07$ 


\section{Introducción}

Las buenas prácticas pedagógicas en matemáticas apoyadas en las TIC, constituyen un reflejo del nivel de competencias que poseen los docentes. El dominio del conocimiento tecnológico como factor fundamental para la consolidación de estas competencias, también contribuye al afianzamiento de un sistema educativo de calidad encaminado a la formación integral de sus educandos. Así como el conocimiento pedagógico y didáctico sobre el uso de las TIC, permite a los docentes mejorar su práctica en el aula y apoyar de forma simultánea la adquisición de las competencias profesionales tanto para los docentes como para los estudiantes (Hernández, Arévalo y Gamboa, 2016); generan posibilidades de cambio y fortalecen los diferentes procesos de la gestión educativa a nivel pedagógico, comunicativo e investigativo (Gamboa, Hernández y Prada, 2018). En el caso de los docentes, algunas de estas tecnologías son estructuradas para el aprendizaje y otras no lo son, pero se adaptan perfectamente al ámbito educativo (Gamboa, Montes y Hernández, 2018).

Se evidencia que el desarrollo de las competencias requeridas para la integración de las TIC en la actividad matemática demanda, por parte del docente, el dominio de diferentes tipos de conocimiento que se ponen en marcha a diario en la práctica pedagógica. Entre estos se determinan el conocimiento pedagógico, el conocimiento disciplinar (en este caso, el conocimiento matemático) y el conocimiento tecnológico. La integración de la tecnología en el proceso de enseñanza y aprendizaje será más eficaz y pertinente de acuerdo al nivel de dominio que tengan los docentes sobre estos conocimientos. Esto permite superar los niveles básicos y tradicionales de asimilación de los distintos constructos matemáticos hacia niveles transformadores e innovadores en las distintas prácticas pedagógicas.

La eficacia en el proceso de enseñanza está supeditado, en buena medida, al sistema de prácticas que el docente desarrolle desde una perspectiva socio-epistémica y cognitiva, en relación con el objeto matemático que aborda. Es decir, que el significado que se le asigna refleja los términos en los que interactúan docente, estudiante y contenido matemático. El tipo de relación que se establece entre ellos incide en las acciones (discursivas y operativas) que conducen a la comprensión y apropiación del conocimiento matemático. Estas acciones también se refieren al eventual uso de recursos tecnológicos que representan un medio más de la variedad de posibilidades existentes para expresar, representar y relacionar un contenido matemático. En este orden de ideas, el dominio epistemológico y procedimental del conocimiento matemático se manifiesta como indicador de la capacidad y disposición del docente para adelantar los procesos de enseñanza y aprendizaje de los contenidos matemáticos.

Sobre los anteriores planteamientos se establecen las siguientes preguntas en torno a las competencias del docente de matemáticas: ¿Poseen un dominio epistemológico adecuado y actualizado de los contenidos que desarrollan en clase?; ¿Muestran competencias digitales suficientes para incorporar la tecnología en el aula como herramienta de apoyo de su práctica pedagógica?; ¿Integran los recursos tecnológicos a las estrategias de enseñanza?; ¿Evalúan los resultados obtenidos al usar la tecnología en el aula y promueven el aprendizaje continuo?; $¿$ Existen diferencias significativas en las valoraciones de las competencias TIC de los docentes de matemáticas, según las características de sus estudiantes (género, edad y carrera que cursan)? A partir de estos interrogantes, se propone como objetivo del presente estudio evaluar las competencias TIC de los docentes de matemáticas según las dimensiones del modelo TPACK (Conocimiento de Contenido, Tecnológico y Pedagógico) desde la perspectiva de los estudiantes al contrastar los dominios de estas competencias en cada dimensión del modelo $\mathrm{y}$ analizar los factores que identifican el perfil académico de los estudiantes. 


\section{EI modelo TPACK}

La matemática es una ciencia mediada por los sistemas semióticos de representación, los cuales tienen la función de transformar la información y generar significados para poder comunicarla (Moreno y Waldegg, 2002). En la actualidad, existe una variedad de instrumentos tecnológicos que permiten a los sujetos elaborar representaciones de los contenidos matemáticos y trabajar las situaciones y problemas desde diferentes perspectivas cognitivas. Estas representaciones resultaban más difíciles de producir mediante el uso de instrumentos tradicionales. Sin embargo, las nuevas tecnologías no resuelven nada por sí solas, por lo tanto es preciso que docentes y estudiantes asuman un rol distinto al del operarios de una máquina y enfrenten de forma racional su uso, orientado al análisis, comprensión e interpretación de los fenómenos de manera integral y conjunta. El resultado de la interacción entre contenido, tecnología y pedagogía permite superar actitudes inertes y mecanicistas que en ocasiones se han manifestado debido al desarrollo fragmentado de la actividad matemática.

En este contexto, autores como Mishra y Koehler, 2006; Koehler y Mishra, 2008; Schmidt, Sahin, Thompson y Seymour, 2008; Schmidt, Baran, Thompson, Mishra, Koehler y Shin, 2009; Koehler, Shin y Mishra, 2012, han formulado el modelo denominado Technological Pedagogical Content Knowledge - TPACK o Conocimiento Tecnológico Pedagógico del Contenido, que destaca la importancia del dominio de tres tipos de conocimientos básicos: tecnológicos, pedagógicos y disciplinares desarrollados por el docente en su actividad profesional y esenciales para la incorporación de la tecnología. El modelo plantea que estos conocimientos no deben considerarse de forma aislada sino que el docente debe interactuar con ellos formando un todo. Esta visión unificadora contribuye a que los profesores aprovechen la tecnología y mejoren el aprendizaje de los estudiantes. Los promotores del TPACK afirman que los profesores tienen que desarrollar su flexibilidad cognitiva de modo que puedan sumar a sus conocimientos, los de los estudiantes, y la información que brinda el contexto, en términos de la escuela, infraestructura disponible y medio ambiente.

\section{Configuración del marco TPACK}

El TPACK está constituido por tres dominios de conocimiento base: el contenido (CK), la pedagogía (PK) y la tecnología (TK). A ellos se suman tres dominios de conocimiento compuestos que resultan de la interacción de las primeras dimensiones: el conocimiento pedagógico de contenido (PCK), el conocimiento tecnológico del contenido (TCK) y el conocimiento tecnológico pedagógico (TPK). La intersección de todos estos dominios conforma el Conocimiento Tecnológico Pedagógico del Contenido - TPACK.

Este modelo ha tenido un fuerte desarrollo en los últimos años. Sin embargo, es preciso reconocer que en lo que se refiere a la formación de los docentes los componentes formulados por Koehler y Mishra (2008) y Mishra y Koehler (2006) no han sido explicados y desarrollados en su totalidad. Autores como Angeli y Valamides (2009) o Cox y Graham (2009) opinan que se requiere de mayor aclaración y especificación. En este sentido, Cox y Graham (2009) llevaron a cabo un estudio con la finalidad de establecer una aclaratoria terminológica de los diferentes elementos del modelo. Para ello, realizaron una revisión conceptual de las definiciones de cada uno de sus componentes, así como una serie de entrevistas a varios investigadores para conocer el significado que le dan a las dimensiones del modelo (PK, CK, TK, PCK, TCK, TPK, y TPACK) y las divergencias existentes entre ellos. En la Tabla 1 se presentan y explican cada una de estas dimensiones.

Estos dominios de conocimiento se encuentran en un estado de equilibrio dinámico, de tensión esencial, de forma tal que un cambio 
Tabla 1.

Dimensiones del modelo TPACK

\begin{tabular}{|c|c|}
\hline Conocimiento & Conceptualización \\
\hline $\begin{array}{l}\text { Conocimiento del Contenido } \\
\text { (CK) }\end{array}$ & $\begin{array}{l}\text { Estos términos hacen referencia al conocimiento que un profesor debe tener sobre los contenidos } \\
\text { que se van aprender o enseñar de una materia o disciplina (Mishra y Koehler, 2006). Los } \\
\text { profesores, además, deben tener conocimiento sobre los hechos que influyen en la enseñanza de } \\
\text { los contenidos, conceptos, teorías y procedimientos de un área específica, así como tener dominio } \\
\text { sobre los marcos explicativos que organizan y conectan el saber específico con los métodos de } \\
\text { demostración propios de la disciplina que enseñan (Shulman, 1986; 1987; 2005). Se trata de } \\
\text { comprender la naturaleza del conocimiento y cómo esta es diferente según cada disciplina. Los } \\
\text { maestros que no cuentan con estos conocimientos pueden tergiversar los temas ante sus alumnos } \\
\text { (Ball y McDiarmid, 1990). Para Gardner (2000), la enseñanza de las disciplinas es el propósito } \\
\text { más importante y menos reemplazable de la escolaridad, ya que proporciona cuatro cosas: el } \\
\text { conocimiento (hechos, conceptos y relaciones), los métodos (creación de conocimiento y procesos } \\
\text { de validación), los propósitos (razones por las cuales existe la disciplina) y por último, las formas } \\
\text { de representación (géneros y sistemas de símbolos). }\end{array}$ \\
\hline $\begin{array}{l}\text { Conocimiento Pedagógico } \\
\text { (PK) }\end{array}$ & $\begin{array}{l}\text { Estos términos designan el conocimiento que deben tener los profesores acerca de los procesos, las } \\
\text { prácticas, métodos de enseñanza-aprendizaje y su manejo con fines y objetivos educativos (Mishra } \\
\text { y Koehler, 2006). Además, incluye el conocimiento acerca de las características socioculturales } \\
\text { y educativas de los estudiantes para la ejecución de estrategias que permitan evaluarlos. Al } \\
\text { maestro debe comprender cómo los estudiantes construyen conocimientos, adquieren habilidades } \\
\text { y desarrollan hábitos mentales También interviene en la gestión del aula, la planificación, la } \\
\text { orientación de las clases, y la evaluación. }\end{array}$ \\
\hline $\begin{array}{l}\text { Conocimiento Tecnológico } \\
\text { (TK) }\end{array}$ & $\begin{array}{l}\text { Es el conocimiento acerca de las tecnologías tradicionales (libros, tiza, pizarra), digitales (Internet, } \\
\text { videos, software) y las habilidades necesarias para su uso. En el caso de las segundas, se incluye } \\
\text { además el conocimiento sobre los sistemas operativos y el hardware de la computadora, así como } \\
\text { la capacidad para utilizar los navegadores web, programas de correo electrónico, procesadores } \\
\text { de texto y hojas de cálculo. También comprende la capacidad del individuo para actualizarse de } \\
\text { forma constante, dado el rápido avance de la tecnología. La adquisición de los conocimientos } \\
\text { tecnológicos permite a una persona procesar la información, comunicarla, resolver problemas y } \\
\text { llevar a cabo una variedad amplia de tareas El conocimiento tecnológico se debe asumir desde su } \\
\text { permanente construcción, que se desarrolla durante toda una vida de interacción y articulación con } \\
\text { la tecnología. }\end{array}$ \\
\hline $\begin{array}{l}\text { Conocimiento Pedagógico } \\
\text { del Contenido (PCK) }\end{array}$ & $\begin{array}{l}\text { Esta dimensión e describe como el resultado de la intersección del conocimiento del contenido y } \\
\text { la pedagogía, esto es, de la relación dependiente y complementaria del uno con respecto a la otra } \\
\text { (Shulman, 1986; 1987; 2005). Desde esta perspectiva, el PCK aborda el conocimiento sobre cómo } \\
\text { determinados temas, problemas o sucesos son organizados, representados, y adaptados según } \\
\text { la diversidad de intereses y capacidades de los estudiantes y luego son presentados de manera } \\
\text { pertinente para su comprensión y aprendizaje (Shulman, 1986). Incluye el conocimiento sobre } \\
\text { las condiciones que promueven el aprendizaje, los vínculos entre el currículo, la evaluación y la } \\
\text { pedagogía, la selección de métodos de enseñanza que se ajustan al contenido y cómo se pueden } \\
\text { organizar estos para una mejor enseñanza. El PCK, también se ocupa de la comprensión de lo que } \\
\text { hace que los conceptos resulten fáciles o difíciles de aprender, de los conocimientos previos de } \\
\text { los estudiantes, de los temas que se deben enseñar y sus formas más útiles de representación, el } \\
\text { uso de analogías, ilustraciones, ejemplos, explicaciones y demostraciones. A partir de la reflexión } \\
\text { e interpretación crítica que el docente realiza sobre la interacción del contenido y la pedagogía, } \\
\text { se lleva a cabo la trasformación del contenido, a través de un ciclo de procesos de comprensión, } \\
\text { transformación, formas de enseñanza, evaluación y reflexión. }\end{array}$ \\
\hline $\begin{array}{l}\text { Conocimiento Tecnológico } \\
\text { del Contenido (TCK) }\end{array}$ & $\begin{array}{l}\text { Es el conocimiento sobre la manera en que la tecnología y el contenido están relacionados (se } \\
\text { influencian y limitan el uno al otro). Los profesores tienen que conocer la forma en que los temas } \\
\text { y las prácticas (o los tipos de representaciones que se pueden construir) pueden cambiar cuando } \\
\text { se emplea la tecnología. También necesitan saber cuáles son las tecnologías más adecuadas para } \\
\text { abordar los contenidos específicos de la materia y cómo estos cambian con el uso de la tecnología, } \\
\text { y viceversa. Comprender que la elección de las tecnologías en algunos casos permite y en otros } \\
\text { restringe el tipo de contenido que se puede enseñar. A su vez, ciertas decisiones sobre el contenido } \\
\text { pueden limitar los tipos de tecnologías que se utilizan en la práctica. La tecnología permite la } \\
\text { construcción de nuevas representaciones al proporcionar un mayor grado de flexibilidad en los } \\
\text { contenidos para su comprensión. Por lo tanto, se sugiere que el uso de la tecnología en el contexto } \\
\text { educativo sea especíico de acuerdo con cada área de conocimiento y según los temas que se } \\
\text { pretende trabajar con estas herramientas (Thompson, 2006). }\end{array}$ \\
\hline
\end{tabular}




\begin{tabular}{|c|c|}
\hline Conocimiento & Conceptualización \\
\hline $\begin{array}{l}\text { Conocimiento Tecnológico } \\
\text { Pedagógico (TPK) }\end{array}$ & $\begin{array}{l}\text { Es el conocimiento sobre la existencia, componentes y potencialidades de las tecnologías. Abarca } \\
\text { la comprensión de su uso en el contexto de enseñanza y aprendizaje, también la variedad de } \\
\text { tecnologías con las que se puede realizar una tarea en particular, la capacidad para elegir una } \\
\text { herramienta con base en la fluidez tecnológica y el conocimiento de estrategias pedagógicas } \\
\text { para el uso de las potencialidades de la tecnología. Incluye el conocimiento sobre herramientas } \\
\text { tecnológicas para llevar a cabo labores propias de la docencia como registros de clase, asistencia, } \\
\text { calificaciones, guías didácticas, socialización de experiencias o paneles de discusión. Dado que } \\
\text { la mayoría de tecnologías están creadas para entornos diferentes al educativo, los docentes deben } \\
\text { demostrar habilidades para reconfigurarlas con fines pedagógicos, con una visión creativa, flexible, } \\
\text { de búsqueda abierta a nuevas soluciones, para promover el aprendizaje. }\end{array}$ \\
\hline $\begin{array}{c}\text { Conocimiento Tecnológico } \\
\text { Pedagógico del Contenido } \\
\text { (TPACK) }\end{array}$ & $\begin{array}{l}\text { Esta dimensión de conocimiento pone énfasis en las conexiones, interacciones, atributos y } \\
\text { restricciones de los elementos del contenido (CK), la pedagogía (PK), y la tecnología (TK). } \\
\text { Esto significa que, además de explorar cada uno de estos dominios de forma aislada, se necesita } \\
\text { observarlos en parejas (PCK, TCK, TPK). En esta visión multidimensional radica el dominio del } \\
\text { conocimiento tecnológico pedagógico del contenido TPACK. La comprensión en conjunto de } \\
\text { estos dominios de conocimiento es central para el desarrollo de una buena enseñanza y práctica } \\
\text { educativa con el uso de la tecnología (Keating y Evans, 2001; Margerum-Leys y Marx, 2002; } \\
\text { Niess, 2005; Zhao, 2003). }\end{array}$ \\
\hline
\end{tabular}

Fuente: Proceso de investigación.

en alguno de ellos tiene que ser compensado por un cambio en los demás (Mishra y Koehler, 2006). En este sentido, el proceso de enseñanza y aprendizaje con tecnología se establece como una relación dinámica entre los componentes señalados. Una visualización aislada de cualquiera de los dominios representa un verdadero perjuicio para la buena enseñanza. Cada situación o problema complejo que se presente en este contexto debe considerarse como una combinación de factores y no debe existir una solución tecnológica única aplicable a cada curso o visión de la enseñanza. Las soluciones se deben encontrar en la capacidad del profesor para navegar con variabilidad en el espacio definido por los tres elementos y las complejas interacciones que se establecen entre estos. Para tal fin, los maestros necesitan desarrollar fluidez y flexibilidad cognitiva no solo en cada uno de los dominios base (TK, PK, y CK), sino también en la manera en que estos dominios se relacionan entre sí, para que se ejecuten soluciones sensibles a contextos educativos específicos.

Esta dimensión del conocimiento (TPACK) invierte la perspectiva convencional de que el contenido solo necesita ser adaptado a una nueva tecnología, o que los objetivos pedagógicos se derivan del uso de la tecno- logía. Cuando se emplean las TIC, los procesos no son tan simples, ya que la integración de una nueva tecnología obliga a enfrentar los problemas educativos básicos y a reconstruir el equilibrio dinámico entre los elementos del programa y la pedagogía en relación con la tecnología. En esta medida el marco TPACK, proporciona el conocimiento necesario para la enseñanza efectiva con tecnología y sobre cómo las tecnologías se utilizan para construir nuevos conocimientos, reconstruir los existentes, desarrollar nuevas métodos o fortalecer los actuales. En esta dimensión de conocimiento, los profesores se deben caracterizar por las formas creativas, flexibles y adaptables con las que deben manejar las limitaciones y posibilidades que ofrecen las herramientas tecnológicas.

Por último, se demuestra la importancia del marco TPACK a partir de la valoración de las acciones formativas emprendidas para la instrucción del docente en TIC, que hacen algunos autores (Marks, 1990; Hu, Walker y Hsiao, 2013; Liu, 2013; Rienties, Brouwer, Bohle, Townsend, Rozendal, Van der Loo y Lygo-Baker., 2013; Mouza, Karchmer-Klein, Nandakumar, Ozden y Hu, 2014). Como ejemplo de estas valoraciones pueden citarse las palabras de Marks (1990), quien señala que: 
[Esta dimensión] representa una clase de conocimiento que es fundamental para el trabajo de los docentes con la tecnología, el cual no está en manos de expertos en la materia con conocimientos tecnológicos, o en técnicos que saben poco del tema o de la pedagogía, o en maestros que saben muy poco de su tema o sobre la tecnología. (p. 9).

\section{Método}

La investigación es de naturaleza cuantitativa. A través del análisis descriptivo-comparativo busca explorar la percepción de los estudiantes frente a las dimensiones básicas de las competencias TIC de los docentes de matemáticas de la Universidad Francisco de Paula Santander. Las dimensiones básicas consideradas desde el marco del modelo TPACK corresponden al conocimiento pedagógico, disciplinar y tecnológico. El desarrollo del proyecto siguió el procedimiento de la investigación por cuestionario, al aplicar un instrumento validado en una muestra representativa de estudiantes de la Universidad en Cúcuta, Colombia. Por tanto, se enmarca dentro de los estudios de campo.

Para su desarrollo se siguieron tres fases:

Adaptación del instrumento para la recogida de los datos. El punto de partida fue la versión original del cuestionario elaborado por Schmidt et al. (2009), traducido después al español y utilizado por Cabero (2014) y validado por Cabero, Marín y Castaño (2015). Para la investigación se rediseñó con la forma de escala tipo Likert. La escala utilizada fue la siguiente: $1=$ totalmente en desacuerdo (TD), 2 = en desacuerdo (D), 3 = ni en desacuerdo ni de acuerdo $(\mathrm{N}), 4=$ de acuerdo $(\mathrm{A}), 5=$ totalmente de acuerdo (TA). La confiabilidad y validez del instrumento se obtuvo al aplicar la técnica de juicio de expertos y la prueba piloto. El valor de Alpha de Cronbach $=0,97$ validó la consistencia interna del instrumento.

Aplicación del instrumento a la muestra seleccionada. Según registros de la Oficina de
División de Sistemas de la Universidad Francisco de Paula Santander, en el momento de la investigación, los planes de estudio abordados alcanzaron los 3980 estudiantes, distribuidos en 136 secciones con un promedio de 32 estudiantes por sección, que cursaron las asignaturas relacionadas con Matemáticas, Cálculo y Algebra. La muestra se estableció siguiendo las fórmulas del muestreo aleatorio simple con un $95 \%$ de confianza y alrededor de un $4 \%$ de error. Este cálculo estableció que la muestra fuera de 589 estudiantes. Su aplicación contó con la colaboración de los docentes adscritos al Departamento de Matemáticas y Estadística de la Universidad y la recolección de los datos se realizó durante el primer semestre académico del año 2015.

Análisis de datos. Esta etapa se desarrolló a través de técnicas de estadística descriptiva, en específico a través del análisis de frecuencias por conjuntos de variables y del análisis de las puntuaciones medias de acuerdo con la escala Likert utilizada. Por otra parte, con el fin de evaluar la presencia de diferencias significativas según el género, la edad y el programa académico cursado, se aplicó el análisis de varianza de un factor (ANOVA). Las variables que orientaron el análisis corresponden a los diferentes tipos de conocimiento base, según el marco del modelo TPACK. Como herramienta de apoyo para realizar este proceso se utilizó el software SPSS versión 23.0.

\section{Resultados y discusión}

\section{Caracterización del grupo de estudiantes encuestados}

La edad de los estudiantes registró una media de 18,88 años con una desviación estándar de 0,09 años y un intervalo de confianza de $95 \%$ para la media que oscila entre 18,69 y 19,08 años. El 50\% de los estudiantes tenía una edad de 18 años o más. El rango de edades fue muy amplio: de 36 años entre el estudiante de menor edad (16 años) y el de mayor (50 años). El 50\% de la distribución de las edades de los 
estudiantes se encuentra entre 18 y 20 años. La distribución del género de los estudiantes por rango de edad se muestra en la Tabla 2. Se observa equilibrio en la proporción de género, pues la muestra estuvo conformada por la mitad de estudiantes de cada género. La mayoría de los estudiantes $(59,8 \%)$ presentaron edades entre 18 y 20 años, cerca de una cuarta parte es menor de 18 años y solo un $1,7 \%$ tiene 25 años o más.

Tabla 2.

Distribución de la muestra por edad y género

\begin{tabular}{|c|c|c|c|c|}
\hline \multirow{2}{*}{ Edad } & \multirow{2}{*}{ Frecuencia } & \multicolumn{2}{|c|}{ Género } & \multirow{2}{*}{ Total } \\
\hline & & Masculino & Femenino & \\
\hline \multirow{2}{*}{$\begin{array}{c}\text { Menores de } \\
18 \text { años }\end{array}$} & $\mathrm{f}$ & 52 & 89 & 141 \\
\hline & $\%$ & 9,1 & 15,6 & 24,7 \\
\hline \multirow{2}{*}{$\begin{array}{c}\text { Entre } 18 \text { y } \\
20 \text { años }\end{array}$} & $\mathrm{f}$ & 179 & 163 & 342 \\
\hline & $\%$ & 31,3 & 28,5 & 59,8 \\
\hline \multirow{2}{*}{$\begin{array}{c}\text { Entre } 21 \text { y } \\
24 \text { años }\end{array}$} & $\mathrm{f}$ & 49 & 30 & 79 \\
\hline & $\%$ & 8,6 & 5,2 & 13,8 \\
\hline \multirow{2}{*}{$\begin{array}{l}25 \text { años o } \\
\text { más }\end{array}$} & $\mathrm{f}$ & 6 & 4 & 10 \\
\hline & $\%$ & 1,0 & 0,7 & 1,7 \\
\hline \multirow{2}{*}{ Total } & $\mathrm{f}$ & 286 & 286 & 572 \\
\hline & $\%$ & 50,0 & 50,0 & 100 \\
\hline
\end{tabular}

Fuente: Proceso de investigación.

La Tabla 3 describe la distribución de la muestra de estudiantes organizados por programa académico y género. Se procuró que la distribución de la muestra fuera representativa en relación con el género, al mantener grupos similares. La mayoría de los estudiantes $(68,7 \%)$ pertenece a los programas académicos de Ingeniería y excede en más del $50 \%$ al otro grupo representado, que son los estudiantes de Administración de Empresas y Contaduría. Se considera que el alcance de la investigación fue significativo porque se incluyeron pequeños grupos de estudiantes de los programas técnicos, del curso preuniversitario y de otros programas académicos. El grupo con mayor representación fue el de los estudiantes de género masculino de la facultad de ingeniería quienes alcanzan el 40\% del grupo.
Tabla 3.

Distribución de los estudiantes por programa académico y género

\begin{tabular}{|c|c|c|c|c|}
\hline \multirow{2}{*}{$\begin{array}{l}\text { Programa } \\
\text { académico }\end{array}$} & \multirow{2}{*}{ Frecuencia } & \multicolumn{2}{|c|}{ Género } & \multirow{2}{*}{ Tota } \\
\hline & & Masculino & Femenino & \\
\hline \multirow{2}{*}{$\begin{array}{c}\text { Administración } \\
\text { de Empresas - } \\
\text { Contaduría }\end{array}$} & $\mathrm{f}$ & 21 & 56 & 77 \\
\hline & $\%$ & 3,6 & 9,6 & 13,2 \\
\hline \multirow{2}{*}{ Ingeniería } & $\mathrm{f}$ & 233 & 167 & 400 \\
\hline & $\%$ & 40,0 & 28,7 & 68,7 \\
\hline \multirow{2}{*}{$\begin{array}{l}\text { Licenciatura } \\
\text { Matemáticas }\end{array}$} & $\mathrm{f}$ & 8 & 9 & 17 \\
\hline & $\%$ & 1,4 & 1,5 & 2,9 \\
\hline \multirow{2}{*}{ Preuniversitario } & $\mathrm{f}$ & 2 & 28 & 30 \\
\hline & $\%$ & 0,3 & 4,8 & 5,2 \\
\hline \multirow{2}{*}{$\begin{array}{l}\text { Programas } \\
\text { Técnicos }\end{array}$} & $\mathrm{f}$ & 20 & 24 & 44 \\
\hline & $\%$ & 3,4 & 4,1 & 7,6 \\
\hline \multirow{2}{*}{$\begin{array}{c}\text { Otros } \\
\text { programas } \\
\text { académicos }\end{array}$} & $\mathrm{f}$ & 6 & 8 & 14 \\
\hline & $\%$ & 1,0 & 1,4 & 2,4 \\
\hline \multirow{2}{*}{ Total } & $\mathrm{f}$ & 290 & 292 & 582 \\
\hline & $\%$ & 49,8 & 50,2 & 100 \\
\hline
\end{tabular}

Fuente: Proceso de investigación.

\section{Análisis descriptivo}

Desde un enfoque descriptivo, a continuación se presenta el informe de lo concerniente a la valoración que hacen los estudiantes sobre los conocimientos base de sus profesores de matemáticas según el marco del modelo TPACK.

\section{Conocimiento pedagógico (PK).}

La Tabla 4 contiene los ítems que miden el conocimiento pedagógico (PK) de los docentes de matemáticas. El $73 \%$ de los estudiantes indican estar de acuerdo en que el docente está atento a las dificultades de los estudiantes para orientarlos oportunamente. Asimismo, el 70\% de los estudiantes manifiesta estar de acuerdo en que los docentes saben cómo evaluar el desempeño de los estudiantes en el aula y solo el $20 \%$ de este grupo cuestiona esta capacidad. Por otra parte, cerca de una tercera parte de los estudiantes cuestiona las competencias de los docentes respecto al conocimiento acerca de 
Tabla 4.

Conocimiento Pedagógico (PK)

\begin{tabular}{|c|c|c|c|c|c|c|c|}
\hline Ítems & Frecuencia & TD & D & ND/NA & $\mathbf{A}$ & TA & Total \\
\hline \multirow{2}{*}{ Sabe cómo evaluar el rendimiento de los estudiantes en el aula. } & $\mathrm{f}$ & 16 & 31 & 71 & 195 & 276 & 589 \\
\hline & $\%$ & 2,7 & 5,3 & 12,1 & 33,1 & 46,9 & 100 \\
\hline \multirow{2}{*}{$\begin{array}{c}\text { Está atento a las dificultades de los estudiantes para entender y } \\
\text { orientarlos oportunamente. }\end{array}$} & $\mathrm{f}$ & 26 & 36 & 94 & 197 & 235 & 588 \\
\hline & $\%$ & 4,4 & 6,1 & 16,0 & 33,5 & 40,0 & 100 \\
\hline \multirow{2}{*}{$\begin{array}{l}\text { Sabe adaptar su estilo de docencia a estudiantes con diferentes } \\
\text { estilos de aprendizaje. }\end{array}$} & $\mathrm{f}$ & 24 & 50 & 137 & 240 & 138 & 589 \\
\hline & $\%$ & 4,1 & 8,5 & 23,3 & 40,7 & 23,4 & 100 \\
\hline \multirow{2}{*}{$\begin{array}{l}\text { Aplica distintas estrategias de evaluación para verificar el proceso } \\
\text { de aprendizaje de los estudiantes. }\end{array}$} & $\mathrm{f}$ & 19 & 45 & 125 & 255 & 145 & 589 \\
\hline & $\%$ & 3,2 & 7,6 & 21,2 & 43,3 & 24,6 & 100 \\
\hline \multirow{2}{*}{$\begin{array}{l}\text { Utiliza una amplia variedad de estrategias de enseñanza y } \\
\text { aprendizaje en el aula (aprendizaje colaborativo, por proyectos, } \\
\text { basado en problemas, etc.). }\end{array}$} & $\mathrm{f}$ & 17 & 41 & 124 & 240 & 167 & 589 \\
\hline & $\%$ & 2,9 & 7,0 & 21,1 & 40,7 & 28,4 & 100 \\
\hline \multirow{2}{*}{ Sabe cómo organizar y mantener la dinámica en el aula. } & $\mathrm{f}$ & 15 & 37 & 133 & 240 & 164 & 589 \\
\hline & $\%$ & 2,5 & 6,3 & 22,6 & 40,7 & 27,8 & 100 \\
\hline \multirow{2}{*}{$\begin{array}{l}\text { Las actividades de enseñanza y aprendizaje que promueve } \\
\text { en el aula favorecen el desarrollo conceptual, actitudinal y } \\
\text { procedimental de los estudiantes. }\end{array}$} & $\mathrm{f}$ & 7 & 40 & 125 & 282 & 135 & 589 \\
\hline & $\%$ & 1,2 & 6,8 & 21,2 & 47,9 & 22,9 & 100 \\
\hline \multirow{2}{*}{$\begin{array}{l}\text { Usa los resultados de la evaluación para el mejoramiento continuo } \\
\text { de los procesos del área. }\end{array}$} & $\mathrm{f}$ & 15 & 38 & 134 & 246 & 156 & 589 \\
\hline & $\%$ & 2,5 & 6,5 & 22,8 & 41,8 & 26,5 & 100 \\
\hline
\end{tabular}

Fuente: Proceso de investigación.

cómo organizar y mantener la dinámica en el aula y usar los resultados de la evaluación para mejorar los procesos del área.

\section{Conocimiento del contenido (CK).}

La Tabla 5 relaciona los ítems que hacen referencia al conocimiento del contenido (CK). Resalta que el $86 \%$ de los estudiantes manifiesta que sus docentes demuestran suficientes conocimientos sobre la materia que enseñan y que asimismo dominan con fluidez el lenguaje propio de su área disciplinar. Por otra parte, hay un conjunto de indicadores en que se cuestiona el conocimiento del contenido (CK) por parte de los docentes que imparten clases de matemáticas. Por ejemplo, el 36\% indica que en raras ocasiones los docentes usan sistemas de símbolos como mediadores en la actividad matemática o científica y alrededor de un $33 \%$ critica la falta de diversidad en el uso de estrategias para desarrollar conocimiento sobre matemáticas o ciencias. De igual modo manifiestan que en pocas oportunidades se plantean situaciones de aula en las que se formulen y resuelvan problemas a partir de situaciones de la vida cotidiana. A pesar de estos cuestionamientos, se destaca el hecho de que más del $70 \%$ de los estudiantes considera que el docente aplica un modo de pensamiento matemático o científico durante el desarrollo de las clases.

\section{Conocimiento tecnológico (TK).}

La Tabla 6 refleja la opinión de los estudiantes ante los indicadores que evalúan el conocimiento tecnológico de los docentes de matemáticas. El 32\% de los estudiantes manifiestan estar de acuerdo en que los docentes poseen las competencias para resolver los problemas técnicos que se presentan en la clase. Cerca del $63 \%$ alude a que el docente asimila conoci- 
Tabla 5.

Conocimiento del Contenido (CK)

\begin{tabular}{|c|c|c|c|c|c|c|c|}
\hline Ítems & Frecuencia & TD & D & ND/NA & $\mathbf{A}$ & TA & Total \\
\hline \multirow{2}{*}{$\begin{array}{l}\text { Demuestra suficientes conocimientos sobre } \\
\text { la materia que enseña. }\end{array}$} & $\mathrm{f}$ & 13 & 23 & 48 & 154 & 351 & 589 \\
\hline & $\%$ & 2,2 & 3,9 & 8,1 & 26,1 & 59,6 & 100 \\
\hline \multirow{2}{*}{ Aplica un modo de pensamiento matemático o científico. } & $\mathrm{f}$ & 12 & 27 & 55 & 184 & 311 & 589 \\
\hline & $\%$ & 2,0 & 4,6 & 9,3 & 31,2 & 52,8 & 100 \\
\hline \multirow{2}{*}{$\begin{array}{l}\text { Representa con facilidad conceptos sobre } \\
\text { matemáticas o ciencias. }\end{array}$} & $\mathrm{f}$ & 13 & 26 & 55 & 193 & 302 & 589 \\
\hline & $\%$ & 2,2 & 4,4 & 9,3 & 32,8 & 51,3 & 100 \\
\hline \multirow{2}{*}{$\begin{array}{l}\text { Interpreta modelos reales que permiten la construcción del } \\
\text { conocimiento sobre matemáticas o ciencias. }\end{array}$} & $\mathrm{f}$ & 15 & 40 & 91 & 192 & 251 & 589 \\
\hline & $\%$ & 2,5 & 6,8 & 15,4 & 32,6 & 42,6 & 100 \\
\hline \multirow{2}{*}{$\begin{array}{l}\text { Usa sistemas de símbolos como mediadores en la actividad } \\
\text { matemática o científica. }\end{array}$} & $\mathrm{f}$ & 16 & 64 & 134 & 256 & 119 & 589 \\
\hline & $\%$ & 2,7 & 10,9 & 22,8 & 43,5 & 20,2 & 100 \\
\hline \multirow{2}{*}{$\begin{array}{c}\text { Conoce varias estrategias para desarrollar conocimiento } \\
\text { sobre matemáticas o ciencias. }\end{array}$} & $\mathrm{f}$ & 17 & 56 & 124 & 233 & 159 & 589 \\
\hline & $\%$ & 2,9 & 9,5 & 21,1 & 39,6 & 27,0 & 100 \\
\hline \multirow{2}{*}{$\begin{array}{l}\text { Domina con fluidez el lenguaje propio } \\
\text { de su área disciplinar. }\end{array}$} & $\mathrm{f}$ & 6 & 15 & 60 & 243 & 265 & 589 \\
\hline & $\%$ & 1,0 & 2,5 & 10,2 & 41,3 & 45,0 & 100 \\
\hline \multirow{2}{*}{$\begin{array}{l}\text { En clase aplica diferentes procedimientos que } \\
\text { permiten resolver situaciones del área. }\end{array}$} & $\mathrm{f}$ & 5 & 36 & 58 & 256 & 234 & 589 \\
\hline & $\%$ & 0,8 & 6,1 & 9,8 & 43,5 & 39,7 & 100 \\
\hline \multirow{2}{*}{$\begin{array}{l}\text { Formula y resuelve problemas a partir de situaciones } \\
\text { de la vida cotidiana. }\end{array}$} & $\mathrm{f}$ & 20 & 39 & 131 & 249 & 150 & 589 \\
\hline & $\%$ & 3,4 & 6,6 & 22,2 & 42,3 & 25,5 & 100 \\
\hline
\end{tabular}

Fuente: Proceso de investigación.

mientos tecnológicos con facilidad y considera que poseen conocimientos actualizados en nuevas tecnologías. Por otra parte, los estudiantes objetan la capacidad de los docentes respecto al conocimiento tecnológico. Por ejemplo, el 57\% cuestiona que el docente haga pruebas con las tecnologías en el aula y casi un 35\% niega que se adopte el uso de distintas tecnologías en el aula. Empero, cerca del 55\% de los estudiantes afirma que sus docentes tienen conocimientos tecnológicos para implementar en el aula.

Al considerar que la escala de acuerdo utilizada es de tipo Likert y que se encuentra en el nivel de intervalo, y que se parte del supuesto de que la distancia entre cada punto o categoría es constante, se presenta de inmediato un resumen del comportamiento de cada ítem en las dimensiones estudiadas con base en su media aritmética (media) y la desviación típi- ca o estándar (DE). La Tabla 7 resume las estadísticas correspondientes a cada ítem. Al ser 3 puntos la media teórica se puede afirmar que todos los ítems tienen una puntuación que va de moderada a alta de acuerdo a la dimensión y el indicador evaluados.

En la Figura 1 se presenta el gráfico de perfiles que resume las dimensiones y permite su comparación. Una valoración global nos permite destacar que el conocimiento del contenido (CK) es superior al pedagógico (PK) y al técnico (TK), mientras que en el nivel inferior aparece el conocimiento tecnológico (TK).

Cuando se examinan los detalles, es posible observar que las medias de los ítems que conforman la dimensión de conocimiento pedagógico (PK) resultaron en general un poco superiores en comparación con las medias de las 
Tabla 6.

Conocimiento Tecnológico TK

\begin{tabular}{|c|c|c|c|c|c|c|c|}
\hline Ítems & Frecuencia & TD & D & ND/NA & $\mathbf{A}$ & TA & Total \\
\hline \multirow{2}{*}{$\begin{array}{l}\text { Resuelve los problemas técnicos } \\
\text { que se le presentan en clase. }\end{array}$} & $\mathrm{f}$ & 58 & 78 & 155 & 190 & 108 & 589 \\
\hline & $\%$ & 9,8 & 13,2 & 26,3 & 32,3 & 18,3 & 100 \\
\hline \multirow{2}{*}{$\begin{array}{l}\text { Se evidencia que asimila conocimientos } \\
\text { tecnológicos fácilmente. }\end{array}$} & $\mathrm{f}$ & 18 & 46 & 150 & 252 & 123 & 589 \\
\hline & $\%$ & 3,1 & 7,8 & 25,5 & 42,8 & 20,9 & 100 \\
\hline \multirow{2}{*}{$\begin{array}{l}\text { Tiene conocimientos actualizados sobre } \\
\text { las nuevas tecnologías. }\end{array}$} & $\mathrm{f}$ & 19 & 46 & 145 & 230 & 149 & 589 \\
\hline & $\%$ & 3,2 & 7,8 & 24,6 & 39,0 & 25,3 & 100 \\
\hline \multirow{2}{*}{ Hace pruebas con la tecnología en clase. } & $\mathrm{f}$ & 73 & 113 & 151 & 168 & 84 & 589 \\
\hline & $\%$ & 12,4 & 19,2 & 25,6 & 28,5 & 14,3 & 100 \\
\hline \multirow{2}{*}{ Usa muchas tecnologías diferentes en clase. } & $\mathrm{f}$ & 80 & 131 & 160 & 152 & 66 & 589 \\
\hline & $\%$ & 13,6 & 22,2 & 27,2 & 25,8 & 11,2 & 100 \\
\hline \multirow{2}{*}{$\begin{array}{l}\text { Demuestra conocimientos al usar } \\
\text { adecuadamente la tecnología. }\end{array}$} & $\mathrm{f}$ & 27 & 54 & 185 & 249 & 74 & 589 \\
\hline & $\%$ & 4,6 & 9,2 & 31,4 & 42,3 & 12,6 & 100 \\
\hline \multirow{2}{*}{$\begin{array}{l}\text { Demuestra experiencia trabajando } \\
\text { con diferentes tecnologías. }\end{array}$} & $\mathrm{f}$ & 26 & 68 & 197 & 206 & 92 & 589 \\
\hline & $\%$ & 4,4 & 11,5 & 33,4 & 35,0 & 15,6 & 100 \\
\hline
\end{tabular}

Fuente: Proceso de investigación.

dimensiones anteriores. Destaca que la mayoría de los estudiantes reporta una alta puntuación relacionada con el conocimiento sobre las estrategias de evaluación de los docentes $(4,16)$ y su capacidad para orientar a los estudiantes que presentan dificultades $(3,98)$. Las medias bajan aproximadamente a 3,70 puntos en la escala cuando se refiere a la capacidad del docente para conocer y adaptar su estilo de enseñanza al estilo de aprendizaje de los estudiantes.

En relación con los ítems que conforman la dimensión conocimiento del contenido (CK), en un plano general los estudiantes reportan que sus docentes poseen un adecuado conocimiento sobre el contenido en todos los indicadores planteados. Los estudiantes otorgan puntajes más altos en los indicadores que hacen referencia al dominio de la materia impartida, a la aplicación de un modo de pensamiento matemático durante la clase y el uso del lenguaje apropia- do a la disciplina. Los elementos que requieren mayor nivel de abstracción, tales como el uso de símbolos, plantean los valores medios más bajos del conjunto $(3,68)$.

Se aprecia que los estudiantes reconocen que sus docentes poseen un nivel alto al considerar con detalle las medias de los ítems que conforman la dimensión de conocimiento tecnológico, pues todos los promedios se ubican por encima de la media teórica equivalente a 3 puntos de la escala; ello, con excepción del ítem que hace referencia al uso de diferentes tecnologías, el cual se encuentra por debajo de 3 (2,99). Sin embargo, los ítems referidos a si los docentes poseen conocimientos y asimilan el conocimiento tecnológico con facilidad, tienden a ubicarse por encima del valor medio teórico de 3 puntos, y alcanzan valores de 3,75 y 3,71 respectivamente. 
COMPETENCIAS TIC DE LOS DOCENTES DE MATEMÁTICAS EN EL MARCO DEL MODELO TPACK:

Tabla 7.

Estadísticos descriptivos para los ítems de las dimensiones de Conocimiento Pedagógico (PK), de Contenido (CK) y Tecnológico (TK)

\begin{tabular}{|c|c|c|c|}
\hline $\begin{array}{l}\text { Número del } \\
\text { ítem }\end{array}$ & Conocimiento Pedagógico (PK) & Media & DE \\
\hline 1 & Sabe cómo evaluar el rendimiento de los estudiantes & 4,16 & 1,010 \\
\hline 2 & Está atento a las dificultades de los estudiantes & 3,98 & 1,097 \\
\hline 3 & Sabe adaptar su estilo de docencia a los estudiantes & 3,71 & 1,044 \\
\hline 4 & Aplica distintas estrategias de evaluación & 3,78 & 1,005 \\
\hline 5 & Utiliza una amplia variedad de estrategias & 3,85 & 1,005 \\
\hline 6 & Sabe cómo organizar y mantener la dinámica en el aula & 3,92 & 0,982 \\
\hline 7 & Las actividades favorecen el desarrollo conceptual & 3,85 & 0,893 \\
\hline 8 & Usa los resultados de la evaluación para el mejoramiento & 3,83 & 0,976 \\
\hline $\begin{array}{l}\text { Número del } \\
\text { ítem }\end{array}$ & Conocimiento del Contenido (CK) & Media & DE \\
\hline 1 & Demuestra suficientes conocimientos & 4,37 & 0,947 \\
\hline 2 & Aplica un modo de pensamiento matemático o científico & 4,28 & 0,954 \\
\hline 3 & Representa con facilidad conceptos & 4,26 & 0,955 \\
\hline 4 & Interpreta modelos reales & 4,06 & 1,039 \\
\hline 5 & Usa sistemas de símbolos & 3,68 & 1,003 \\
\hline 6 & Conoce varias estrategias para desarrollar conocimiento & 3,78 & 1,037 \\
\hline 7 & Domina con fluidez el lenguaje propio de su área & 4,27 & 0,822 \\
\hline 8 & En clase aplica diferentes procedimientos & 4,15 & 0,892 \\
\hline 9 & Formula y resuelve problemas & 3,80 & 1,004 \\
\hline $\begin{array}{l}\text { Número del } \\
\text { ítem }\end{array}$ & Conocimiento Tecnológico (TK) & Media & DE \\
\hline 1 & Resuelve los problemas técnicos & 3,36 & 1,206 \\
\hline 2 & Se evidencia que asimila conocimientos & 3,71 & 0,983 \\
\hline 3 & Tiene conocimientos actualizados & 3,75 & 1,021 \\
\hline 4 & Hace pruebas con la tecnología en clase & 3,13 & 1,236 \\
\hline 5 & Usa tecnologías diferentes en clase & 2,99 & 1,214 \\
\hline 6 & Demuestra conocimientos & 3,49 & 0,980 \\
\hline 7 & Demuestra experiencia trabajando con tecnologías & 3,46 & 1,029 \\
\hline
\end{tabular}

Fuente: Proceso de investigación. 


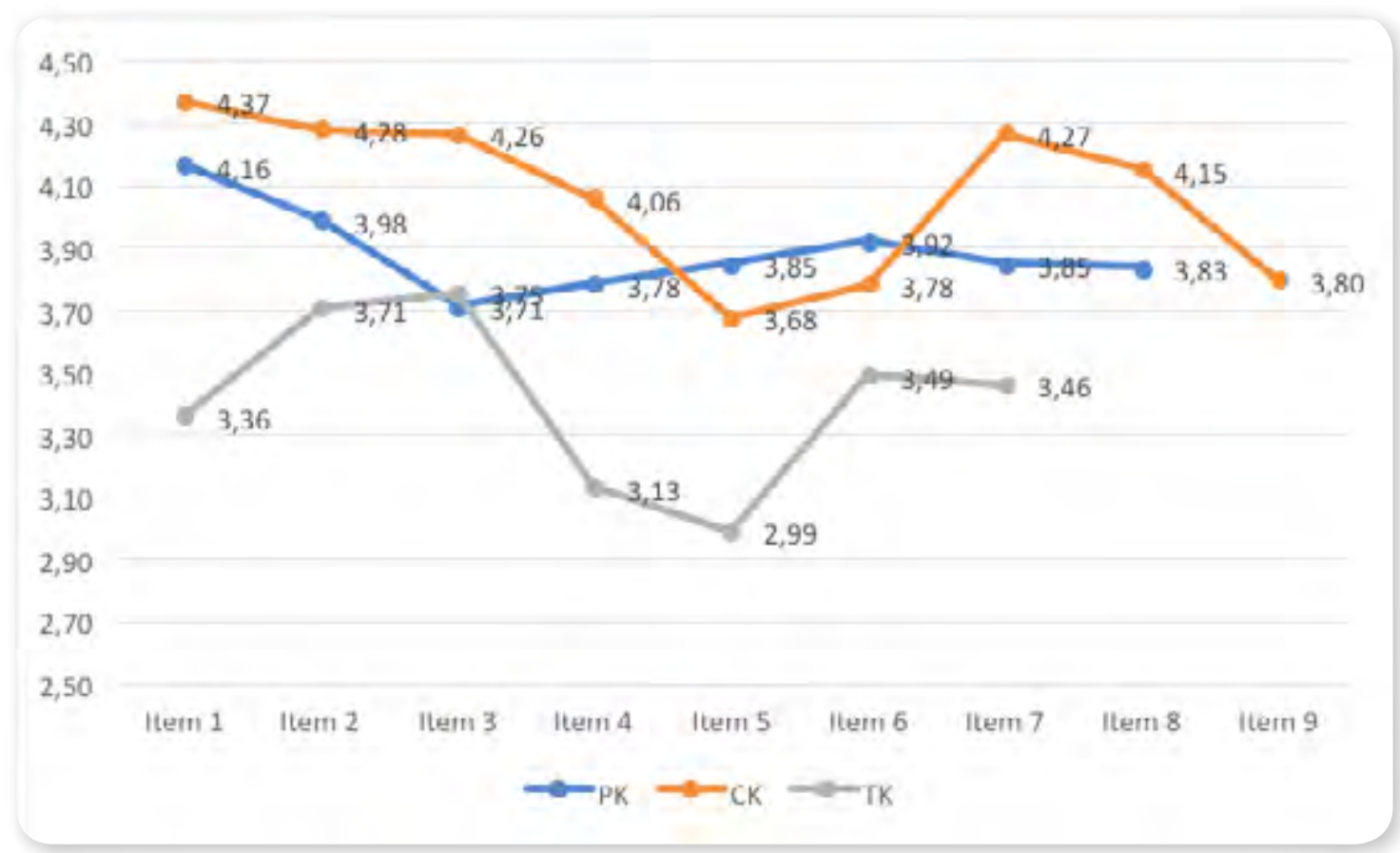

Figura 1. Gráfico de perfiles sobre las medias de las dimensiones de Conocimiento Pedagógico, (PK), de Contenido (CK) y Tecnológico (TK). Fuente: Proceso de investigación.

\section{Análisis comparativo con base en las puntuaciones totales.}

Los estadísticos descriptivos para las puntuaciones totales de la escala en cada una de las dimensiones analizadas pueden observarse en la Tabla 8. Con respecto a la variabilidad, las medidas son muy homogéneas y la desviación estándar se encuentra entre el rango de 5,6 y 5,9 puntos. Las medias, aunque son muy variables, no son comparables en primera instancia porque los ítems que conforman cada escala son distintos.

La Tabla 9 muestra a través de un análisis de varianza ANOVA una comparación de los niveles medios de cada una de estas puntuaciones considerando como factores el género, el rango de edad y el programa académico. En cuanto a género, no existen diferencias signi- ficativas en los totales medios de los tipos de conocimientos, pues todos los valores de $\mathrm{p}$ son mayores que el nivel de significación de 0,05 ( $>0,05)$. Considerando los rangos de edad (especificados), las diferencias tampoco resultaron significativas al nivel del 5\%.

La Tabla 10 muestra el ANOVA de un factor al tener en cuenta como variable independiente el programa académico que cursan los estudiantes. Se evidencian diferencias importantes en cuanto a las dimensiones del conocimiento tecnológico y de contenido, $\mathrm{p}=0,00<0,05 \mathrm{y} p=0,04<0,05$ Esto significa que las valoraciones medias en esta dimensión difieren de acuerdo con el programa académico que cursan los estudiantes, pero no ocurre lo mismo con el conocimiento pedagógico, en el que no se registraron diferencias representati$\operatorname{vas}(\mathrm{p}=0,13>0,05)$. 
A partir de la Tabla 11 se puede profundizar en estas diferencias. En ella se observa que el conocimiento tecnológico en el programa de Licenciatura en Matemáticas presenta las valoraciones más bajas, con 18 puntos, seguido de otros programas académicos con 21 puntos y de Ingeniería con 23 puntos. Por otra parte, y de forma paradójica, los estudiantes de Licencia- tura en Matemáticas y de Ingeniería son los que mejor valoran el conocimiento del contenido de sus docentes, de lo cual se desprende que, aunque no consideran que posean competencias tecnológicas suficientes en el aula, sí valoran su preparación profesional y dominio del contenido que ellos imparten.

Tabla 8.

Estadísticos descriptivos para las puntuaciones totales de las dimensiones básicas del TPACK

\begin{tabular}{|l|r|r|r|r|r|r|r}
\hline $\begin{array}{c}\text { Dimensiones base - } \\
\text { Conocimiento TPACK }\end{array}$ & \multicolumn{1}{|c|}{ Media } & \multicolumn{1}{c|}{ DE } & Med & \multicolumn{1}{c|}{ Máx } & \multicolumn{1}{c|}{ Mĺn } & P25 & \multicolumn{1}{c}{ P75 } \\
\hline Conocimiento Tecnológico & 23,89 & 5,63 & 24,00 & 35,00 & 7,00 & 20,00 & 28,00 \\
\hline Conocimiento de Contenido & 36,65 & 5,98 & 38,00 & 45,00 & 9,00 & 33,00 & 41,00 \\
\hline Conocimiento Pedagógico & 31,01 & 5,81 & 32,00 & 40,00 & 10,00 & 28,00 & 35,00 \\
\hline
\end{tabular}

Fuente: Proceso de investigación.

Tabla 9.

Resultados del ANOVA de un factor para las puntaciones totales según el género

\begin{tabular}{|l|r|r|r}
\multicolumn{1}{c|}{ Dimensiones base conocimiento } & $\begin{array}{c}\text { Suma de } \\
\text { cuadrados }\end{array}$ & F & Sig. \\
\hline Conocimiento Tecnológico & 4,966 & 0,156 & 0,693 \\
\hline Conocimiento de Contenido & 120,979 & 3,375 & 0,067 \\
\hline Conocimiento Pedagógico & 25,216 & 0,746 & 0,388
\end{tabular}

Fuente: Proceso de investigación.

Tabla 10.

Resultados del ANOVA de un factor para las puntaciones totales según el programa académico

\begin{tabular}{|c|c|c|c|}
\hline $\begin{array}{c}\text { Dimensiones base conocimiento } \\
\text { TPACK }\end{array}$ & $\begin{array}{l}\text { Suma de } \\
\text { cuadrados }\end{array}$ & F & Sig. \\
\hline Conocimiento Tecnológico & 851,938 & 5,580 & 0,000 \\
\hline Conocimiento de Contenido & 410,007 & 2,318 & 0,042 \\
\hline Conocimiento Pedagógico & 281,040 & 1,677 & 0,138 \\
\hline
\end{tabular}

Fuente: Proceso de investigación. 
Tabla 11.

Puntuaciones totales medias por dimensión según los programas académicos cursados

\begin{tabular}{|c|c|c|c|}
\hline $\begin{array}{l}\text { Dimensiones } \\
\text { TPACK }\end{array}$ & Programa académico & Media & DE \\
\hline \multirow{6}{*}{$\begin{array}{l}\text { Conocimiento } \\
\text { Tecnológico }\end{array}$} & Administración de Empresas - Contaduría & 24,09 & 5,04 \\
\hline & Ingeniería & 23,91 & 5,62 \\
\hline & Licenciatura Matemáticas & 18,00 & 7,08 \\
\hline & Preuniversitario & 25,80 & 5,20 \\
\hline & Programas Técnicos & 25,14 & 4,88 \\
\hline & Otros programas académicos & 21,71 & 5,82 \\
\hline \multirow{6}{*}{$\begin{array}{l}\text { Conocimiento } \\
\text { Contenido }\end{array}$} & Administración de Empresas - Contaduría & 35,93 & 6,78 \\
\hline & Ingeniería & 36,94 & 5,76 \\
\hline & Licenciatura Matemáticas & 38,59 & 3,64 \\
\hline & Preuniversitario & 37,40 & 5,30 \\
\hline & Programas Técnicos & 35,30 & 6,48 \\
\hline & Otros programas académicos & 33,47 & 7,48 \\
\hline \multirow{6}{*}{$\begin{array}{l}\text { Conocimiento } \\
\text { Pedagógico }\end{array}$} & Administración de Empresas - Contaduría & 30,76 & 6,78 \\
\hline & Ingeniería & 31,24 & 5,36 \\
\hline & Licenciatura Matemáticas & 33,41 & 4,14 \\
\hline & Preuniversitario & 29,23 & 6,65 \\
\hline & Programas Técnicos & 30,18 & 7,30 \\
\hline & Otros programas académicos & 29,65 & 5,87 \\
\hline
\end{tabular}

Fuente: Proceso de investigación.

\section{Conclusiones}

Con respecto a los conocimientos base, en primer lugar los estudiantes perciben que sus docentes de matemáticas poseen un mayor dominio del conocimiento disciplinar (CK), seguido del conocimiento pedagógico (PK) y por último, sobresale el dominio del conocimiento tecnológico (TK). En cuanto al dominio del conocimiento disciplinar (matemático), destacan la aplicación de este conocimiento durante la práctica educativa y señalan la facilidad para representar los conceptos y para usar de manera fluida el lenguaje propio de las matemáticas. Sin embargo, en esta dimensión los estudiantes perciben de sus docentes dificultad para usar sistemas de símbolos como mediadores en la actividad matemática.
En segundo lugar se encuentra el conocimiento pedagógico, en el que se destaca la capacidad de los docentes para usar estrategias que permiten evaluar el aprendizaje de los estudiantes y la destreza para orientarlos de forma oportuna de acuerdo con las dificultades que se les presentan durante el desarrollo de los contenidos matemáticos. Las falencias tienen que ver con mantener la organización, la dinámica del aula y en el reconocimiento de los diferentes estilos de aprendizaje para adaptar su práctica. Finalmente, con un promedio bajo de aceptación se encuentra el conocimiento tecnológico, en el que se percibe que los docentes tienen conocimientos suficientes sobre la tecnología para usarla apropiadamente en contextos particulares. Aun así, los estudiantes cuestionan sus competencias para usar diferentes tipos de tecnología en el contexto del aula. 
En relación con el dominio de los conocimientos compuestos denominados Conocimiento Pedagógico del Contenido, Conocimiento Tecnológico del Contenido y Conocimiento Tecnológico Pedagógico (PCK, TCK, TPK), se determina que existe un desarrollo desigual entre ellos. Por una parte, existe un dominio coherente sobre los competentes relacionados con los procesos de enseñanza del conocimiento disciplinar (PCK) y la aplicación de este conocimiento en diferentes contextos. Así como, su relación con diferentes tipos de herramientas que permiten la mediación en este proceso y facilitan el acceso a este campo de conocimiento (TPK). Sin embargo, el desequilibrio se evidencia cuando se incorpora la tecnología al dominio del contenido disciplinar (TCK). En este caso, se cuestiona sobre la falta de prácticas de aula en donde predomine el dominio de los procesos matemáticos como la demostración, conceptualización, modelación a través de la mediación de la tecnología. En este caso, se desarrollan procesos de comprensión, análisis y desarrollo procedimental de contenidos sin el uso de recursos tecnológicos.

En cuanto al conocimiento TPACK, los estudiantes destacan que los docentes utilizan y aplican su conocimiento tecnológico actual para dar solución a los problemas propios del área cuando se les presentan. Sin embargo, cuestionan sus competencias para llevar a cabo procesos de integración de la tecnología, con los métodos de enseñanza y los contenidos matemáticos, seleccionar las tecnologías adecuadas para orientar los contenidos y para usar de manera flexible y adaptable las limitaciones que traen consigo estas herramientas.

En este contexto, se evidencia una relación dispar entre las dimensiones de conocimiento del marco TPACK. El desequilibrio entre los elementos que conforman los tipos de conocimientos base con los conocimientos relacionados del marco TPACK define para los docentes de matemáticas un nivel de conocimiento tecnológico pedagógico disciplinar moderado bajo, tal y como lo señalaron también Mishra y Koehler (2006). Al respecto, se determina que el conocimiento matemático y pedagógico de los docentes les permite desarrollar prácticas educativas acordes a las necesidades curriculares que plantea cada programa académico para una formación competente. Sin embargo, el desarrollo de competencias matemáticas a través de la mediación tecnológica requiere de parte del docente capacitación, diseño-planeación, control y seguimiento de su práctica educativa, con el fin de alcanzar de manera eficiente y exitosa el proceso de enseñanza y aprendizaje de las matemáticas.

\section{Referencias}

Angeli, C., y Valanides, N. (2009). Epistemological and methodological issues for the conceptualization, development, and assessment of ICT-TPCK: Advances in technological pedagogical content knowledge (TPCK). Computers y Education, 52(1), 154-168. doi: 10.1016/j.compedu.2008.07.006

Arévalo Duarte, M. A. (2016). Competencias TIC de los docentes en matemáticas en el marco del modelo TPACK. Una perspectiva para el desarrollo de buenas prácticas pedagógicas. [Tesis de doctorado]. Universidad de Salamanca, España.

Ball, D. L., y McDiarmid, W. (1990). The Subject-Matter Preparation of Teachers. En W. R. Houston (Ed.), Handbook for Research on Teacher Education. New York, United States: Macmillan.

Cabero, J. (Dir.) (2014). La formación del profesorado en TIC: Modelo TPACK (Conocimiento Tecnológico, Pedagógico y de Contenido). Sevilla: Secretariado de Recursos Audiovisuales de la Universidad de Sevilla - Publidisa S.A.

Cabero, J., Marín, V., y Castaño, C. (2015). Validación de la aplicación del modelo TPACK para la formación del profeso- 
rado en TIC. @tic.revista d'innovació educativa, 14, 13-22. doi: 10.7203/attic. 14.4001

Cox, S., y Graham, C. R. (2009). Diagramming TPACK in practice: Using an elaborated model of the TPACK framework to analyze and depict teacher knowledge. TechTrends: Linking Research y Practice to Improve Learning, 53(5), 60-69. doi: 10.1007/s11528-009-0327-1

Gamboa,A.,Hernández,C.A.,yPrada,R.(2018). Práctica pedagógica y competencias TIC: atributos y niveles de integración en docentes de instituciones educativas de básica y media. Saber, Ciencia y Libertad, 13(1), 258-274. doi: 10.18041/2382-3240/ saber.2018v13n1.2090

Gamboa, A.; Montes, A., y Hernández, C.A. (2018). Representaciones de los docentes de educación básica sobre los aportes de las Tecnologías de la Información y la Comunicación en la escuela. Revista ESPACIOS, 39(2), 2.

Gardner, H. (2000). Disciplined mind: beyond facts and standardized tests, the k-12 education that every child deserves. New York, United States: Penguin Putnam.

Hernández, C. A., Arévalo, M. A., y Gamboa, A. A. (2016). Competencias TIC para el desarrollo profesional docente en educación básica. Praxis y Saber, 7(14), 41-69. doi: 10.19053/22160159.5217

Hu, H-W., Walker, K., y Hsiao, W-Y. (2013). Developing elementary pre-service teachers' technological, pedagogical, and content knowledge for learning and teaching division of fractions. International Journal of Technology, Knowledge and Society, 9(2), 185-204. doi: 10.18848/1832-3669/ CGP/v09i02

Keating, T., y Evans, E. (2001). Three computers in the back of the classroom: preservice teachers' conceptions of technology integration. En J. Price, D. Willis, N. Davis y J. Willis (Eds.), Proceedings of Society for Information Technology \& Teacher Education International Conference 2001 (pp. 1671-1676). Chesapeake, VA, United States: Association for the Advancement of Computing in Education (AACE).

Koehler, M. J., y Mishra, P. (2008). Introducing TPCK. En AACTE Committee on Technology and Innovation (Eds.), Handbook of technological pedagogical content knowledge (TPCK) for educators (pp. 3-29). New York, United States: Routledge

Koehler, M. J., Shin, T. S., y Mishra, P. (2012). How do we measure TPACK? Let me count the ways. En Educational technology, teacher knowledge, and classroom impact: A research handbook on frameworks and approaches (pp. 16-31). IGI Global. doi: 10.4018/978-1-60960-750-0. ch002

Liu, S-H. (2013). Exploring the Instructional Strategies of Elementary School Teachers. Intemational Education Studies, 6(11), 58-68. doi: 10.5539/ies.v6n11p58

Margerum-Leys, J., y Marx, R. (2002). Teacher knowledge ol educational technology: A case study of student teacher/mentor teacher pairs. Journal of Educational Computing Research, 26(4), 427-462. doi: 10.2190/JXBR-2G0G-1E4T-7T4M

Marks, R. (1990). Pedagogical content knowledge: From a mathematical case to a modified conception. Journal of Teacher Education, 41(3), 3-11. doi: $10.1177 / 002248719004100302$

Mishra, P., y Koehler, M. J. (2006). Technological Pedagogical Content Knowledge: A Framework for Teacher Knowledge. Teachers College Record, 108(6), 1017-1054. doi: 10.1111/j.1467-9620.2006.00684 
Moreno, L., y Waldegg, G. (2002). Fundamentación cognitiva del currículo de matemáticas. En: Seminario Nacional de Formación de Docentes: Uso de Nuevas Tecnologías en el aula de matemáticas (pp. 40-66). Bogotá, Colombia: Ministerio de Educación de Colombia.

Mouza C., Karchmer-Klein R., Nandakumar R., Ozden S. Y., y Hu L. (2014). Investigating the impact of an integrated approach to the development of preservice teachers' technological pedagogical content knowledge (TPACK). Computers y Education 71, 206-221. doi: 10.1016/j. compedu.2013.09.020

Niess, M. L. (2005). Preparing teachers to teach science and mathematics with technology: Developing a technology pedagogical content knowledge. Teaching and Teacher Education, 21(5), 509-523. doi: $10.1016 /$ j.tate.2005.03.006

Rienties, B., Brouwer, N., Bohle Carbonell, K., Townsend, D., Rozendal, A-P., van der Loo, J., y Lygo-Baker, S. (2013). Online training of TPACK skills of higher education scholars: A cross-institutional impact study. European Journal of Teacher Education, 36(4), 480-495. doi: $10.1080 / 02619768.2013 .801073$

Schmidt, D., Baran, E., Thompson, A., Mishra, P., Koehler, M. J., y Shin, T. (2009). Technological pedagogical content knowledge (TPACK): The development and validation of an assessment instrument for preservice teachers. Journal of Research on Technology, 42(2), 123-149. doi: $10.1080 / 15391523.2009 .10782544$
Schmidt, D., Sahin, E. B., Thompson, A., y Seymour, J. (2008). Developing effective technological pedagogical and content knowledge (TPACK) in preK-6 teachers. En K. McFerrin, R. Weber, R. Carlsen y D. A. Willis (Eds.), Proceedings of the Society for Information Technology $y$ Teacher Education International Conference 2008 (pp. 5313-5317). Chesapeake: AACE.

Shulman, L. (1986). Those who understand: Knowledge growth in teaching. Educational Researcher, 15(2), 4-14. doi: 10.3102/0013189x015002004

Shulman, L. S. (1987). Knowledge and Teaching: Foundations of the new reform. Harvard Educational Review, 57(1), 1-22. doi: 10.17763/haer.57.1.

Shulman, L.S. (2005). Conocimiento y enseñanza: fundamentos de la nueva reforma. Profesorado. Revista de currículum y formación del profesorado, 9(2), 1-30.

Thompson, A. (2006). Technology pedagogical content knowledge: Framing teacher knowledge about technology. Journal of Computing in Teacher Education, 22(6), 46-48.

Zhao, Y. (Ed.) (2003). What should teachers know about technology? Perspectives and practices. Greenwich, CO: Information Age Publishing. 
